\title{
Regulation of antimycin biosynthesis by the orphan ECF RNA polymerase sigma factor $\sigma$ AntA
}

Antimycins are an extended family of depsipeptides that are made by filamentous actinomycete bacteria and were first isolated more than 60 years ago. Recently, antimycins have attracted renewed interest because of their activities against the anti-apoptotic machineries inside human cells which could make them promising anti-cancer compounds. The biosynthetic pathway for antimycins was recently characterised but very little is known about the organisation and regulation of the antimycin (ant) gene cluster. Here we report that the ant gene cluster in Streptomyces albus is organized into four transcriptional units; the antBA, antCDE, antGF and antHIJKLMNO operons. Unusually for secondary metabolite clusters, the antG and antH promoters are regulated by an extracytoplasmic function (ECF) RNA polymerase sigma factor named $\sigma^{\text {AntA }}$ which represents a new sub-family of ECF $\sigma$ factors that is only found in antimycin producing strains. We show that $\sigma^{\text {AntA }}$ controls production of the unusual precursor 3-aminosalicylate which is absolutely required for the

production of antimycins. $\sigma^{A n t A}$ is highly conserved in antimycin producing strains and the -10 and -35 elements at the $\sigma^{A n t A}$ regulated ant $G$ and $a n t H$ promoters are also highly conserved suggesting a common mechanism of regulation. We also demonstrate that altering the Cterminal Ala-Ala residues found in all $\sigma^{\text {AntA }}$ proteins to Asp-Asp increases expression of the antFG and antGHIJKLMNO operons and we speculate that this Ala-Ala motif may be a signal for the protease CIpXP. 
1 Regulation of antimycin biosynthesis by the orphan ECF RNA polymerase sigma factor $\sigma^{\text {AntA }}$ 2

3 Ryan F. Seipke ${ }^{1,2 *}$, Elaine Patrick ${ }^{1}$ and Matthew I. Hutchings ${ }^{1 *}$

4

$5{ }^{1}$ School of Biological Sciences, University of East Anglia, Norwich Research Park, Norwich,

$6 \quad$ NR4 7TJ, United Kingdom

7

$8{ }^{2}$ Current address: The Faculty of Biological Sciences, University of Leeds, Leeds, LS2 9JT

9 United Kingdom

10

11 *For correspondence E-mail: $\underline{\text { m.hutchings@uea.ac.uk or r.seipke@leeds.ac.uk }}$

12 


\section{INTRODUCTION}

Approximately $60 \%$ of the antibiotics and anticancer compounds currently used in human medicine are derived from the secondary metabolites of soil-dwelling Streptomyces species and other filamentous actinomycetes. Although the vast majority of these natural products were discovered more than 40 years ago, the advent of genome mining and new tools to unlock socalled "silent" pathways mean that these bacteria still offer us the best hope of developing new antibiotics for clinical use. The antimycin family of natural products were discovered nearly 65 years ago and initially attracted interest because of their potent antifungal activity (Dunshee et a1., 1949). Antimycins are widely produced by Streptomyces species and they exhibit a range of bioactive properties, including antifungal, insecticidal and nematocidal activity. This is the result of their ability to inhibit cytochrome c reductase, an enzyme in the respiratory chain in bacteria and mitochondria. Antimycins are also used as piscicides (brand name Fintrol ${ }^{\circledR}$ ) to kill off unwanted scaled fish in the farming of catfish, which are relatively insensitive to antimycins (Finlayson et al., 2002). More recently antimycins have been shown to be potent and selective inhibitors of the mitochondrial Bcl-2/Bcl- $\mathrm{x}_{\mathrm{L}}$-related anti-apoptotic proteins which are overproduced by drug resistant cancer cells. Over-production of $\mathrm{Bcl}-2 / \mathrm{Bcl}-\mathrm{x}_{\mathrm{L}}$ proteins in cancer cells confers resistance to multiple chemotherapeutic agents whose primary mode of action is to trigger apoptosis. Antimycins bind to the hydrophobic groove of Bcl-2-type proteins and inhibit their activity in a mechanism of action that is independent of their activity against electron transport (Tzung et al., 2001). A synthetic derivative of antimycin $\mathrm{A}_{3}, 2$-methoxyantimycin $\mathrm{A}_{3}$ (2MeAA), no longer inhibits the respiratory chain, but retains potent antagonistic activity toward Bcl-2-related proteins and induces apoptosis (Tzung et al., 2001; Schwartz et al., 2007). This has led to suggestions that antimycin derivatives such as 2-MeAA could be used alongside traditional apoptosis-inducing chemotherapeutics to block drug resistance and kill cancer cells. There is significant interest in bioengineering antimycins with improved pharmacological properties for the treatment of cancer and infectious diseases.

Despite their unique chemical structure and important biological properties, the antimycin biosynthetic pathway was only reported very recently (Seipke et al., 2011a,b) and rapid progress has been made in elucidating the biosynthetic steps in this pathway over the last two years (for a recent review see Seipke and Hutchings, 2013). Antimycins are produced by a hybrid nonribosomal peptide synthetase (NRPS) / polyketide synthase (PKS) assembly line for which the 44 complete biosynthetic pathway has been proposed (Sandy et al., 2012; Yan et al., 2012). The 45 AntFGHIJKLN proteins encode the biosynthetic pathway for the unusual starter unit, 3- 
aminosalicylate-CoA (Schoenian et al., 2012; Sandy et al., 2012). The AntCD proteins comprise the hybrid NRPS / PKS machinery, and AntE and AntM are crotonyl-CoA reductase and discrete ketoreductase homologues, respectively (Sandy et al., 2012). AntO and AntB are tailoring enzymes. AntO is predicted to install the $N$-formyl group (Yan et al., 2012; Sandy et al., 2012), and AntB is a promiscuous acyltransferase that catalyses a transesterification reaction of a hydroxl group at C-8 to result in the acyloxyl moiety and the chemical diversity observed at R1 (Sandy et al., 2013). The antA gene encodes an extracytoplasmic function (ECF) RNA polymerase sigma $(\sigma)$ factor named $\sigma^{\text {AntA }}$ which, like all other ECF $\sigma$ factors, contains only two of the four $\sigma^{70}$ domains (Staron et al. 2009).

The resurgence of interest in the biosynthesis of antimycins and particularly in engineering new analogues with better pharmacological properties led us to investigate the transcriptional organisation and regulation of the antimycin gene cluster. The only regulator encoded by the ant gene cluster is $\sigma^{\text {AntA }}$, but regulation of secondary metabolite clusters by ECF $\sigma$ factors is unusual and has not yet been reported in Streptomyces species. To our knowledge only two examples of ECF $\sigma$ factor regulation of antibiotic biosynthesis have been described and both differ from $\sigma^{\text {AntA }}$ because they are co-encoded with, and regulated by, anti- $\sigma$ factors whereas $\sigma^{\text {AntA }}$ is an orphan, i.e. it has no co-encoded anti- $\sigma$ factor. The two known examples both control lantibiotic production in rare actinomycetes. In Microbospora corallina, the pathway specific regulator MibR and the ECF $\sigma^{\mathrm{MibX}}$ regulate microbisporicin biosynthesis and $\sigma^{\mathrm{MibX}}$ is regulated by MibW (Foulston and Bibb, 2010). In Planomonospora alba the pathway specific regulator PspR, the ECF sigma factor $\sigma^{\mathrm{PspX}}$ and its anti- $\sigma$ factor PspW all regulate production of the lantibiotic planosporicin (Sherwood and Bibb, 2013). The closest homologues to $\sigma^{\mathrm{MibX}}$ and MibW are $\sigma^{\mathrm{PspX}}$ and its anti- $\sigma$ factor PspW, suggesting a common mechanism of regulation for these lantibiotics.

Here we characterize the gene organization of the antimycin gene cluster and the role of $\sigma^{\text {AntA }}$ in Streptomyces albus $\mathrm{S} 4$. We report that $\sigma^{\text {AntA }}$ is regulated at the transcriptional level and controls production of the unusual precursor 3-aminosalicylate that is required for antimycin production. We also show that $\sigma^{\text {AntA }}$ represents a new sub-family of ECF $\sigma$ factors that are only found in the ant gene clusters of Streptomyces species and provide evidence that suggests $\sigma^{\text {AntA }}$ regulation of the divergent antGF and antHIJKLMNO operons is conserved in all antimycin producing strains. Finally we provide preliminary evidence that the activity of $\sigma^{\text {AntA }}$ is affected by the two C-terminal amino acid residues such that altering the natural Ala-Ala residues to Asp-Asp increases expression of the $\sigma^{\text {AntA }}$ target genes. Since a C-terminal Ala-Ala motif is a well known signal for the serine protease ClpXP (Flynn et al. 2003) this may provide a novel post- 
translational mechanism for controlling $\sigma^{\text {AntA }}$ activity without the need for an anti- $\sigma$ factor.

\section{Materials and Methods}

Growth media and strains. Streptomyces strains (Table 1) were grown on mannitol-soya flour (MS) agar and Lennox broth (LB) (Kieser et al., 2000), and Escherichia coli strains (Table 1) were grown on LB or LB agar. Growth media was supplemented with antibiotics as required at the following concentrations: apramycin $(50 \mu \mathrm{g} / \mathrm{ml})$, carbenicillin $(100 \mu \mathrm{g} / \mathrm{ml})$, hygromycin B (50 $\mu \mathrm{g} / \mathrm{ml})$, kanamycin $(50 \mu \mathrm{g} / \mathrm{ml})$, nalidixic acid $(50 \mu \mathrm{g} / \mathrm{ml})$. All Streptomyces strains were created using cross-genera conjugation in which DNA was transferred from E. coli ET12567/pUZ8007 (MacNeil et al., 1992) according to standard methods (Kieser et al., 2000).

Cosmid library construction and screening. A Supercos1 cosmid library was constructed from Streptomyces albus S4 genomic DNA partially digested with Sau3AI and packaged into Gigapack III XL phage according to the manufacturer's instructions (Agilent Technologies). One thousand cosmid clones were screened by PCR using primers RFS172 and RFS173 (Table S2), which target an internal fragment of the antC gene. Cosmid 456 and cosmid 213 tested positive and were end-sequenced using primers RFS184 and RFS185 (Table S2) and mapped onto the Streptomyces albus S4 genome using BLAST 2.2.23+ (Altschul et al., 1990).

\section{Construction of Streptomyces albus S4 mutant strains. Mutant strains were constructed} using $\lambda$-RED based PCR-targeting mutagenesis (Gust et al., 2003). A disruption cassette consisting of a conjugal origin of transfer (oriT) and the apramycin resistance gene, aac(3)IV from pIJ773 (Gust et al., 2003), was generated by PCR using BioTaq polymerase (Bioline) and oligonucleotide primers (Table S2) containing $39 \mathrm{nt}$ of homology that included the start and stop

101 codons of each gene (with the exception of the STRS4_02213-02217 multi-mutant) and $36 \mathrm{nt}$ 102 upstream or downstream of the open reading frame. The resulting PCR products were gel purified 103 and electroporated into E. coli BW25113/pIJ790 harboring either cosmid 456 (ASTRS4_02194,

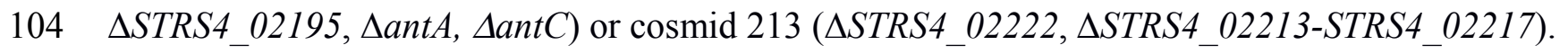

105 Transformants were screened for the presence of mutagenised cosmid by Not I digestion.

106 Mutagenised cosmids were moved to S. albus S4 by conjugation. Transconjugants were selected

107 for apramycin resistance and kanamycin sensitivity. The integrity of mutant strains was verified

108 by PCR using flanking primers for each deleted coding sequence together and in combination 109 with the P1 and P2 primers which target the apramycin cassette (Gust et al., 2003). Combinations 110 RRF228 and 229, RRF278 and 279 and RRF329 and 330 were used to test the 02194,02195 and 
11102212 knockouts, respectively (Table S2).

112 Construction of plasmids. In order to heterologously express and purify AntA, the antA

113 coding sequence was PCR-amplified from genomic DNA using oligonucleotide primers

114 engineered to possess NdeI and HindIII restriction sites (RFS230 and RFS 231, Table S2) using

115 Phusion polymerase (New England Biolabs). The resulting PCR product was gel purified and

116 digested with NdeI and HindIII (Roche) and ligated with pET28a (Novagene) cut with the same

117 enzymes using T4 DNA ligase (Promega) to create pET28a-antAI. DNA sequencing using the T7

118 promoter and $\mathrm{T} 7$ terminator primers (Novagene) verified the integrity of the cloned ant $A$ coding

119 sequence. In order to construct the antA over-expression / complementation plasmid, pIJ10257-

120 antA, the antA coding sequence was excised from pET28a-antA using NdeI and HindIII and

121 ligated to pIJ10257 (Hong et al., 2005) cut with the same enzymes. pIJ10257-antA was

122 introduced into Streptomyces strains by conjugation and transconjugants were selected for

123 resistance for hygromycin.

124 In order to generate complementation constructs in which transcription of wild-type and

125 mutated antA was initiated by its native promoter, we replaced the antB gene with an apramycin

126 resistance cassette using the REDIRECT system described above using oligos RFS188 and

127 RFS189 (Gust et al., 2003, Table S2). The apramycin cassette possesses two FRT sites recognised

128 by the FLP recombinase. The mutagenised cosmid was introduced into E. coli strain BT340,

129 which expresses a FLP recombinase when cultured at $42{ }^{\circ} \mathrm{C}$ (Gust et al., 2003). FLP

130 recombinase-mediated excision of the apramycin resistance cassette leaves an $81 \mathrm{bp}$ in-frame

131 “scar." Cosmid 213 santB-flp was used as template for PCR with the forward primer RFS351

132 and the reverse primers RFS231 or RFS352 (Table S2). RFS351 targets 270 bp upstream of the

133 putative $a n t B$ start codon, and RFS231 and RFS352 both target an identical sequence in the C-

134 terminus of antA, with the exception that RFS352 introduces two C->A point mutations, which

135 introduces A172D and A173D changes into the resulting AntA protein. These PCR products were

136 cloned into pGEMT-Easy (Promega) and verified by DNA sequencing using M13R and M13F

137 oligonucleotides. Next, the antA-containing inserts were excised from pGEMT-Easy by EcoRI

138 digestion and ligated with pAU3-45 (Bignell et al., 2005) digested with the same enzyme. pAU3-

139 45-antA-AA and pAU3-45-antA-DD were introduced into Streptomyces strains by conjugation

140 and transconjugants were selected for resistance to thiostrepton.

142 Phylogenetic analysis. Antimycin gene clusters were analysed from S. ambofaciens ATCC 23877 
143 (AM238663, (Choulet et al., 2006)), S. blastmyceticus NBRC 12747 (AB727666, (Yan et al.,

144 2012)), S. gancidicus BKS 13-15 [AOHP00000000, (Kumar et al., 2013), S. griseoflavus Tü4000

145 (ACFA00000000), S. hygroscopicus subsp.jinggangensis 5008 (NC_017765), S. hygroscopicus

146 subsp.jinggangensis TL01 (NC_020895), Streptomyces sp. 303MFCol5.2 (ARTR00000000),

147 Streptomyces sp. TOR3209 (AGNH00000000, (Hu et al., 2012), S. albus S4 (CADY00000000,

148 (Seipke et al., 2011b)), S. albus J1074 (NC_020990), Streptomyces sp. SM8 (AMPN00000000),

149 Streptomyces sp. NRRL2288 (JX131329), (Yan et al., 2012)), Streptomyces sp. LaPpAH-202

150 (ARDM00000000), Streptomyces sp. CNY228 (ARIN01000033). $\sigma^{\text {AntA }}$ proteins were aligned to

151 five (when possible) random proteins from each ECF RNA polymerase $\sigma$ factor subfamily

152 defined by Staron et al (Staron et al., 2009) by using Clustal $\Omega$ (Sievers et al., 2011). The

153 phylogenetic tree was created using PhyML 3.0 with the default settings (Guindon et al., 2010)

154 and visualised using FigTree v1.4 (http://tree.bio.ed.ac.uk/software/figtree/).

HPLC analysis. Wild-type and mutant strains were cultured atop a cellophane disc on MS

156 agar. At the time of harvest, the cellophane disc containing mycelia was removed and either

157 processed for RNA extraction (below) or discarded. Bacterial metabolites were extracted from the

158 spent agar using $50 \mathrm{ml}$ of ethyl acetate for 1 hour. $20 \mathrm{ml}$ of ethyl acetate was evaporated to

159 dryness under reduced pressure and the resulting residue was resuspended in $400 \mu 1100 \%$

160 methanol. In all cases, the methanolic extracts from at least two biological replicates were mixed

161 and centrifuged at $>16,000 \mathrm{~g}$ in a microcentrifuge prior to analysis. Antimycin $\mathrm{A}_{1}-\mathrm{A}_{4}$ standards

162 were purchased from Sigma-Aldrich. 35 microliters of methanolic extract was separated on a

163 Phenomenex C18(2) $5 \mu \mathrm{m} 4.6$ x $150 \mathrm{~mm}$ using a Hitachi L-6200 HPLC system and the following

164 gradient (solvent A: water, solvent B: methanol, flow rate $1 \mathrm{ml} / \mathrm{min}$ ): 0-20 min, 10-100\% B; 20-

$16534 \min 100 \%$ B; 34.1-44 min, 10\% B. Samples were analysed with a Shimadzu M20A Photo

166 Diode Array.

167 RNA analysis. For all experiments involving RNA, S. albus S4 strains were cultivated at

$16830^{\circ} \mathrm{C}$ on MS agar atop a cellophane disc to facilitate the easy harvest of mycelia into

169 microcentrifuge tubes. Transcription was arrested using a stop solution (95\% ethanol, 5\% acid

170 phenol) diluted 1:4 with water. Total RNA was extracted using a RNeasy Mini Kit (Qiagen)

171 according to the manufacturer's instructions and included both an on-column and a post-column

172 DNaseI treatment. The absence of DNA contamination was assessed by PCR. DNase-treated

173 RNA was reverse transcribed using $250 \mu \mathrm{g}$ of random hexamers and Superscript III reverse

174 transcriptase (RT, Invitrogen) with an extension temperature of $55^{\circ} \mathrm{C}$. 
For co-transcription analysis, twenty-nine cycles of PCR amplification with six primer

176 sets (Table S2) were performed using cDNA originating from $5 \mu \mathrm{g}$ of RNA with BioTaq

177 Polymerase (Bioline). Primer sets were designed to span the intergenic regions of the antimycin

178 cluster and targeted at least $300 \mathrm{bp}$ upstream of putative start codons to account for promoters

179 driving transcription from multiple sites within a transcriptional unit. RNA from the

180 complemented antA mutant strain ( $\triangle a n t A / \mathrm{pIJ} 10257-$ antA $)$ was used, because transcript

181 abundance was greater for operons involved in 3-aminosalicylate biosynthesis. The PCR products

182 obtained were cloned into either pCRII-TOPO (Invitrogen) or pGEM-T Easy (Promega) and

183 sequenced by either the Genome Analysis Centre (Norwich, UK), Source BioScience

184 (Cambridge, UK), or Eurofins MWG Operon (Ebersberg, Germany) using oligonucleotide primer

185 M13r (Integrated DNA Technologies).

186 For quantitative RT-PCR, gene-specific primers were designed to amplify $\sim 100 \mathrm{bp}$ from

187 the first and last gene of each transcriptional unit in the antimycin cluster. cDNA was diluted (1

188 volume of cDNA to 2 volumes of water) and target genes were quantified using a Bio-Rad

189 CFX96TM instrument and SensiFast ${ }^{\mathrm{TM}}$ SYBR No-ROX kit (Bioline). Each treatment consisted

190 of three biological replicates and two technical replicates. The calculated $\mathrm{C} t$ (threshold cycle

191 value) for each target gene was normalized to the $\mathrm{C} t$ obtained for the $h r d B$ gene, which encodes

192 the vegetative sigma factor and is routinely used as a reference gene for transcriptional analyses

193 (Kelemen et al., 1996).

194 For mapping of transcriptional start sites, $10 \mu \mathrm{g}$ of RNA from the $\Delta$ ant $A / \mathrm{pIJ} 10257$-antA

195 strain was processed using the FirstChoice ${ }^{\circledR}$ RLM-RACE Kit (Ambion) according to the

196 manufacturer's instructions with the following modifications: for cDNA synthesis, Superscript III

197 RT (Invitrogen) was used to according the manufacturer's instructions using an extension

198 temperature of $55^{\circ} \mathrm{C}$. The gene-specific primers used for each transcriptional unit are listed in

199 Table S2. The final PCR products were gel purified and cloned into pCRII-TOPO (Invitrogen) or

200 pGEM-T Easy (Promega) and sequenced using oligonucleotide primers M13r (Integrated DNA

201 Technologies) by either the Genome Analysis Centre (Norwich, UK), Source BioScience

202 (Cambridge, UK) or Eurofins MWG Operon (Ebersberg, Germany). The transcriptional start site

203 was determined to be the nucleotide immediately adjacent to the sequence of the 5'RLM-RACE

204 RNA adapter.

205 Bacterial two-hybrid analysis. The full STRS4_02195, AntA, and STRS4_04339 (SigB

206 orthologue) coding sequences were PCR-amplified from S4 genomic DNA using Phusion 
207 Polymerase (New England Biolabs) and primers RFS280 and RFS281 (STRS4_02195), RFS282

208 and RFS283 (antA), and sigB (RFS284 and RFS285) (Table S1). The gel purified PCR products

209 were digested with BamHI and KpnI (Roche) and cloned into bacterial two-hybrid plasmids

210 pUT18C and pKT25 (Karimova et al., 1998) cut with the same enzymes. Cloned inserts were

211 sequenced by The Genome Analysis Centre (Norwich, UK) using primers RFS286, RFS287

212 (pUT18C clones) and RFS288 and RFS289 (pKT25 clones) to ensure that no mutations had

213 occurred. Plasmid combinations of interest were co-electroporated into E. coli DHM1 and

214 processed for $\beta$-galactosidase activity as previously described (Hutchings et al., 2002).

216 Results and Discussion

217 Organisation and expression of the antimycin gene cluster. To facilitate mutagenesis of the

218 antimycin gene cluster, we constructed a Supercos1 library of the S. albus S4 genome (Genbank

219 accession CADY00000000.1) and screened the library by PCR against an internal fragment of

220 antC. We identified two overlapping cosmids containing antC and confirmed that cosmid 213

221 contains the complete predicted ant gene cluster by deleting genes adjacent to the cluster using

222 PCR-targeted mutagenesis (Fig. 1). To define the upstream border we deleted STRS4_02194,

223 which encodes a separate NRPS and STRS4_02195 which encodes a predicted membrane protein

224 of unknown function. To determine the downstream border we deleted STRS4_02212 and

225 STRS4_02214-STRS4_02217 which are predicted to encode a nitrate / nitrite assimilation protein

226 and an $\mathrm{ABC}$-transport system, respectively. To determine if these mutations affect antimycin

227 production we performed bioassays against the human pathogen Candida albicans and observed

228 no obvious difference in the ability of the S. albus S4 strains to inhibit the growth of C. albicans

229 compared to wild-type (Fig. 2A). High performance liquid chromatography (HPLC) confirmed

230 that antimycin production is not affected by any of these mutations showing that STRS4_02194,

231 STRS4_02195,STRS4_02212, and STRS4_02214-02217 mark the boundaries of the ant gene

232 cluster (Fig. 2B). The gene organization of the ant cluster suggests there is a minimum of four

233 transcriptional units with the largest being the antHIJKLMNO operon (Fig 1). Almost all of these

234 ORFs overlap, suggesting transcriptional and translational coupling, but as a proof of principle

235 we confirmed that the antGF and antHIJKLMNO genes are co-transcribed by performing end-

236 point RT-PCR. Six primer pairs were designed to span the intergenic (or overlapping gene)

237 regions of the antGF and antHIJKLMNO operons and targeted at least $300 \mathrm{bp}$ upstream of the

238 putative start codons to detect transcriptional read-through. Six PCR products were obtained by 
RT-PCR analysis and sequenced to confirm that antGF and antHIJKLMNO form two operons.

240 No products were obtained when reverse transcriptase was omitted (Fig. S1). In addition to

241 confirming that antGF and antHIJKLMNO are organized into operons, this also validates our

242 approach to analysing their expression using qRT-PCR to measure mRNA levels of the first and

243 last genes in each operon.

244 Streptomyces species have a complex life cycle that includes growth as a substrate

245 mycelium that gives rise to aerial mycelia and sporulation. To determine at which stage of the life

246 cycle the antimycin gene cluster is expressed we measured expression of the four ant operons

247 after 18 and 42 hours growth on mannitol-soya flour (MS) agar. After 18 hours growth on MS

248 agar S. albus $\mathrm{S} 4$ consists entirely of substrate mycelium but after 42 hours the substrate mycelium

249 has differentiated to produce aerial mycelium and spores. All four ant operons are expressed at a

250 significantly higher level at 18 hours (in substrate mycelium) compared to 42 hours which

251 suggests that all four ant operons are switched off following differentiation (Fig. 3A).

252 Conversely, HPLC analysis of mycelium and culture medium extracted at the same time points

253 only detected antimycins in the 42 hour samples suggesting there is a lag between ant gene

254 expression and antimycin production (Fig 3B). This is probably due to the time it takes for the

255 precursor to be produced and for the antimycin scaffold to be assembled and then accumulate to

256 detectable levels. Most notably, these data suggest that specific regulatory mechanisms exist to

257 activate ant gene expression in substrate mycelium and switch it off again following

258 differentiation. Since antA is the only putative regulatory gene in the ant gene cluster we

259 investigated the role of $\sigma^{\text {AntA }}$ in regulating antimycin production.

Antimycin production is dependent on the orphan ECF sigma factor $\sigma^{\text {AntA }}$. To investigate the

262 role of $\sigma^{\text {AntA }}$ in regulating antimycin biosynthesis, we deleted the antA gene and tested the mutant

263 strain against $C$. albicans in a bioassay. The antA mutant is significantly less active against $C$.

264 albicans compared to wild-type and this is consistent with loss of antimycin production (Seipke

265 et al., 2011a). Complementation of this mutant with the antA gene under the control of the strong

266 constitutive ermE* promoter restores bioactivity against C. albicans to wild-type levels (Fig. 4A)

267 and HPLC analysis confirmed that antimycins are not produced by the antA mutant (Fig. 4B). We

268 conclude that $\sigma^{\text {AntA }}$ is required for antimycin production

269 To determine which of the four ant promoters are regulated by $\sigma^{\text {AntA }}$ we used qRT-PCR to

270 measure ant operon expression in the wild-type and antA strains grown for 18 hours on MS agar.

271 Deletion of antA did not affect the level of transcription of either the antBA or antCDE operons, 
272 but transcription of both the antGF and antHIJKLMNO operons was significantly reduced in the 273 antA mutant (Fig. 5). This suggests that $\sigma^{\text {AntA }}$ positively regulates the transcription of the 274 antFGHIJKLMNO genes which encode biosynthesis of 3-aminosalicylate, the precursor used by 275 the AntC NRPS. Furthermore, over-expression of $\sigma^{\text {AntA }}$ in 42 hour cultures activates the 276 expression of the antGF and antHIJKLMNO operons leading us to conclude that no additional 277 regulators are required to activate the $a n t G$ and ant $H$ promoters (Fig. 6). In addition the $a n t B$ and 278 ant $C$ promoters must be regulated by a transcription factor encoded outside of the ant gene 279 cluster since they are upregulated at 18 hours relative to 42 hours growth. To confirm this we 280 introduced cosmid 213 into $S$. lividans, $S$. coelicolor M145 and the $S$. coelicolor superhost strains 281 M1146, M1152 and M1154 (Gomez-Escribano and Bibb, 2011) but failed to detect antimycin 282 production, supporting the idea that at least one additional transcription activator is required.

$\sigma^{\text {AntA }}$ and its putative binding site are highly conserved. Bioinformatic analysis failed to identify the common ECF $\sigma$ factor promoter motifs upstream of the ant $G$ and ant $H$ genes, notably the "AAC" motif in the -35 region and the "CGT" motif in the -10 region (Staron et al., 2009). We therefore mapped the transcriptional start sites of the antGF and antHIJKLMNO operons using 5'-RLM RACE and identified -10 and -35 regions which share high nucleotide sequence identity with one another, but not with the $\sigma^{\text {AntA }}$-independent $a n t B$ promoter (Fig. 7A). Six antimycin producing Streptomyces strains have been reported previously (Riclea et al., 2012; Seipke et al., 2011a; Yan et al., 2012) and we identified eight more putative antimycin gene clusters whilst searching for $\sigma^{\mathrm{Ant} A}$ orthologues in Genbank (Table S1 and Experimental Procedures). Since the 14

294 known $\sigma^{\text {AntA }}$ orthologues share 66\% sequence identity (Table S1, Fig. S2), we hypothesise that

$295 \sigma^{\text {AntA }}$ regulation of the ant $G$ and $a n t H$ promoters will be common to all antimycin producing 296 Streptomyces strains. To investigate this, we searched for the ant $G$ and ant $H$ promoter motifs in 297 the 14 known or predicted antimycin gene clusters encoded by published Streptomyces genome 298 sequences. All 14 ant $G$ promoters contain very high sequence identity in the -35 and -10 regions, 299 although $S$. ambofaciens has an 18 nucleotide spacer between the -35 and -10 element compared 300 to the typical 17 nucleotide spacer (Fig. 7B). High nucleotide conservation was also observed at 301 the antH promoter and the -10 element contains a "CTC" motif that is $100 \%$ conserved across all 302 promoters although again spacer regions between the -10 and -35 elements vary in length 303 between 17 and 18 bp (Fig. 7B). The in silico data therefore suggests that $\sigma^{\text {AntA }}$ has highly 304 conserved -35 and -10 binding sites at the ant $G$ and ant $H$ promoters of all antimycin producing 
305 Streptomyces strains. Scanning the complete published S. albus genome with the AntA -10 and

$306-35$ binding sites (using GLEME2 - part of MEME) returns only two significant hits, the antGF

307 and antHIJKLMNO promoters suggesting there are no other $\sigma^{\text {AntA }}$ targets (results not shown). This

308 strongly suggests that $\sigma^{\text {AntA }}$ is a pathway-specific regulator of antimycin biosynthesis.

309

$310 \sigma^{A n t A}$ represents a new sub-family of ECF sigma factors. $\sigma^{\text {AntA }}$ contains only the $\sigma_{2}$ and $\sigma_{4}$

311 domains (Pfam families PF04542 and PF08281) which is characteristic of the ECF family of

312 RNA polymerase $\sigma$ factors (Staron et al., 2009). However, $\sigma^{\text {AntA }}$ does not fit into any of the ECF

313 sub-families listed in the well-maintained public database ECF Finder (Staron et al., 2009).

314 Multiple sequence alignments of the $14 \sigma^{\text {AntA }}$ homologues in the database and representatives of

315 all known ECF sub-families revealed that the $\sigma^{\text {AntA }}$ proteins form a distinct clade and therefore

316 represent a new sub-family of ECF $\sigma$ factors (Table S1 and Fig. S3). ECF $\sigma$ factors are rare in

317 secondary metabolite gene clusters and to our knowledge this is the first example in Streptomyces

318 species (Foulston and Bibb, 2010; Sherwood and Bibb, 2013). The only obvious candidate for an

319 anti- $\sigma^{\text {AntA }}$ factor in the antimycin gene cluster is the putative membrane protein STRS4_02195.

320 However, it is absent from the ant clusters in other streptomycetes, its removal has no effect on

321 antimycin biosynthesis (Fig. 2) and it does not interact with $\sigma^{\text {AntA }}$ in bacterial two-hybrid analysis

322 (Fig S4) which leads us to conclude that $\sigma^{\text {AntA }}$ is an orphan ECF that is not subject to anti- $\sigma$ factor

323 control. However, since antA expression is activated in substrate mycelium (by an as yet

324 unknown regulator) and switched off following differentiation we predict that a mechanism might

325 exist to remove $\sigma^{\text {AntA }}$ protein at this stage of growth. The only unusual feature in the primary

326 sequence of the $14 \sigma^{\text {AntA }}$ homologues is the conserved C-terminal Ala-Ala (AA) motif (Fig. S2)

327 which is a known signal for the serine protease ClpXP (Flynn et al., 2003). To test whether the C-

328 terminal AA residues are required for $\sigma^{\text {AntA }}$ activity we made two identical constructs expressed

329 under the control of the native antB promoter. The first construct drives production of the wild-

330 type protein (designated $\sigma^{\text {AntA-AA }}$ ) and the second drives production of an altered protein in which

331 the AA has been replaced with DD (designated $\sigma^{\text {AntA-DD }}$ ). We introduced these constructs into the

332 antA mutant and measured expression of the antGF and antHIJKLMNO operons in these strains.

333 Both operons are significantly more highly expressed in the strain producing $\sigma^{\text {AntA-DD }}$ compared

334 with the wild type $\sigma^{\text {AntA-AA }}$ protein (Fig. 8). These data suggest that the two C-terminal residues

335 play an important role in the stability and / or activity of $\sigma^{\text {AntA }}$ and may target $\sigma^{\text {AntA }}$ for proteolysis

336 by ClpXP. Unfortunately, all attempts to detect the $\sigma^{\text {AntA }}$ protein by immunoblotting whole cell

337 extracts with polyclonal anti- $\sigma^{\text {AntA }}$ antibodies have been unsuccessful while tagging the protein at 
338 the N-terminus inactivates the protein (not shown). Future work will therefore be required to

339 determine the role of the C-terminal AA motif in $\sigma^{\text {AntA }}$ however, we have provided preliminary

340 evidence that suggests $\sigma^{\text {AntA }}$ might be a direct target for ClpXP, thereby bypassing the requirement

341 for the additional level of anti- $\sigma$ regulation. This would also provide a rapid mechanism to shut

342 down precursor biosynthesis when antimycins are no longer required.

344 Acknowledgments.

345 We thank Charles Brearley for assistance with HPLC, Barrie Wlikinson, Mervyn Bibb and Mark

346 Buttner for helpful comments on this work and all members of the Hutchings group and the UEA

347 iGem 2013 team for useful discussions.

\section{REFERENCES}

350 Altschul SF, Gish W, Miller W, Myers EW and Lipman DJ. (1990). Basic local alignment search 351 tool. Journal of Molecular Biology 215: 403-410.

352 Barke J, Seipke RF, Gruschow S, Heavens D, Drou N, Bibb MJ, Goss RJM, Yu DW and

353 Hutchings MI (2010). A mixed community of actinomycetes produce multiple antibiotics for the

354 fungus farming ant Acromyrmex octospinosus. BMC Biology 8: 109.

355 Bignell DRD, Tahlan K, Colvin KR, Jensen SE and Leskiw BK. (2005). Expression of ccaR,

356 encoding the positive activator of cephamycin C and clavulanic acid production in Streptomyces

357 clavuligerus, is dependent on bldG. Antimicrobial Agents and Chemotherapy 49: 1529-1541.

358 Choulet F, Aigle B, Gallois A, Mangenot S, Gerbaud C, Truong C, Francou FX, Fourrier C,

359 Gurineau M, Decaris B, Barbe V, Pernodet JL and LeBlond P. (2006). Evolution of the terminal

360 regions of the Streptomyces linear chromosome. Molecular Biology and Evolution 23: 2361-

3612369.

362 Crooks GE, Hon G, Chandonia JM and Brenner SE. (2004). WebLogo: A sequence logo

363 generator. Genome Research 14: 1188-1190

364 Dunshee BR, Leben C, Keitt GW and Strong FM. (1949). The isolation and properties of

365 antimycin A. Journal of the American Chemical Society 71: 2436-2437.

366 Finlayson BJ, Schnick RA, Cailteux RL, DeMong L, Horton WD, McClay W and Thompson

367 CW. (2002). Assessment of Antimycin a Use in Fisheries and its Potential for Reregistration.

368 Fisheries 27: 10-18.

369 Flynn JM, Neher SB, Kim YI, Sauer RT and Baker TA. (2003). Proteomic discovery of cellular

370 substrates of the ClpXP protease reveals five classes of ClpX-recognition signals. Molecular Cell, 
11: $671-683$.

372 Foulston LC and Bibb MJ. (2010). Microbisporicin gene cluster reveals unusual features of

373 lantibiotic biosynthesis in actinomycetes. Proceedings of the National Acadamy of Sciences USA

374 107: 13461-13466.

375 Guindon S, Dufayard JF, Lefort V, Anisimova M, Hordijk W and Gascuel O. (2010). New

376 Algorithms and Methods to Estimate Maximum-Likelihood Phylogenies: Assessing the

377 Performance of PhyML 3.0. Systematic Biology 59: 307-321.

378 Gust B, Challis GL, Fowler K, Kieser T and Chater KF. (2003). PCR-targeted Streptomyces gene

379 replacement identifies a protein domain needed for biosynthesis of the sesquiterpene soil odor

380 geosmin. Proceedings of the National Acadamy of Sciences USA 100: 1541-1546.

381 Hong H-J, Hutchings MI, Hill LM and Buttner MJ. (2005). The role of the novel Fem protein

382 VanK in vancomycin resistance in Streptomyces coelicolor. Journal of Biological Chemistry

383 280: 13055-13061.

384 Hu D, Li X, Chang Y, He H, Zhang C, Jia N, Li H and Wang Z. (2012). Genome Sequence of

385 Streptomyces sp. Strain TOR3209, a Rhizosphere Microecology Regulator Isolated from Tomato

386 Rhizosphere. Journal of Bacteriology 194: 1627-1627.

387 Hutchings MI, Crack JC, Shearer N, Thompson BJ, Thomson AJ and Spiro S. (2002).

388 Transcription factor FnrP from Paracoccus denitrificans contains an iron-sulfur cluster and is

389 activated by anoxia: identification of essential cysteine residues. Journal of Bacteriology 184:

$390 \quad 503-508$.

391 Karimova G, Pidoux J, Ullmann A and Ladant D. (1998). A bacterial two-hybrid system based on

392 a reconstituted signal transduction pathway. Proceedings of the National Acadamy of Sciences

393 USA 95: 5752-5756.

394 Kelemen GH, Brown GL, Kormanec J, Potúcková L, Chater KF and Buttner MJ. (1996). The

395 positions of the sigma-factor genes, whiG and $\operatorname{sig} F$, in the hierarchy controlling the development

396 of spore chains in the aerial hyphae of Streptomyces coelicolor A3(2). Molecular Microbiology

397 21: 593-603.

398 Kieser T, Bibb MJ, Buttner MJ, Chater KF and Hopwood DA. (2000). Practical Streptomyces

399 Genetics. Norwich: The John Innes Foundation.

400 Kumar S, Kaur N, Singh NK, Raghava GPS and Mayilraj S. (2013). Draft Genome Sequence of

401 Streptomyces gancidicus Strain BKS 13-15. Genome Announcements 1: e00150-13.

402 MacNeil DJ, Gewain KM, Ruby CL, Dezeny G, Gibbons PH and MacNeil T. (1992). Analysis of

403 Streptomyces avermitilis genes required for avermectin biosynthesis utilizing a novel integration 
404 vector. Gene 111: 61-68.

405 Riclea R, Aigle B, Leblond P, Schoenian I, Spiteller D. and Dickschat J.S. (2012). Volatile

406 Lactones from Streptomycetes Arise via the Antimycin Biosynthetic Pathway. ChemBioChem 13:

407 1635-1644.

408 Sandy M, Rui Z, Gallagher J and Zhang W. (2012). Enzymatic Synthesis of Dilactone Scaffold of 409 Antimycins. ACS Chemical Biology 7: 1956-61.

410 Sandy M, Zhu X, Rui Z and Zhang W. (2013). Characterization of AntB, a Promiscuous

411 Acyltransferase Involved in Antimycin Biosynthesis. Organic Letters 15: 3396-9.

412 Schoenian I, Paetz C, Dickschat JS, Aigle B, Leblond P and Spiteller D. (2012). An

413 unprecedented 1,2-shift in the biosynthesis of the 3-aminosalicylate moiety of antimycins.

$414 \quad$ Chembiochem 13: 769-73.

415 Seipke RF, Barke J, Brearley C, Hill L, Yu DW, Goss RJM and Hutchings MI. (2011a). A single

416 Streptomyces symbiont makes multiple antifungals to support the fungus farming ant

417 Acromyrmex octospinosus. PLoS ONE, 6: e22028.

418 Seipke RF, Crossman L, Drou N, Heavens D, Bibb MJ, Caccamo M and Hutchings MI. (2011b).

419 Draft Genome Sequence of Streptomyces Strain S4, a Symbiont of the Leaf-Cutting Ant

420 Acromyrmex octospinosus. Journal of Bacteriology 193: 4270-4271.

421 Seipke RF and Hutchings MI. (2013). The regulation and biosynthesis of antimycins. Beilstein J

422 Org Chem. 9: 2556-2563.

423 Sherwood EJ and Bibb MJ. (2013). The antibiotic planosporicin coordinates its own production

424 in the actinomycete Planomonospora alba. Proceedings of the National Acadamy of Sciences

425 USA 110: E2500-9.

426 Schwartz PS, Manion MK, Emerson CB, Fry JS, Schulz CM, Sweet IR and Hockenbery DM.

427 (2007). 2-Methoxy antimycin reveals a unique mechanism for Bcl-xL inhibition. Molecular

428 Cancer Therapeutics 6: 2073-2080.

429 Sievers F, Wilm A, Dineen D, Gibson TJ, Karplus K, Li W, Lopez R, McWilliam H, Remmert M,

430 Soding J, Thompson JD and Higgins DG. (2011). Fast, scalable generation of high-quality

431 protein multiple sequence alignments using Clustal Omega. Molecular Systems Biology 7: 1-6.

432 Staron A, Sofia HJ, Dietrich S, Ulrich LE, Liesegang H and Mascher T. (2009). The third pillar of

433 bacterial signal transduction: classification of the extracytoplasmic function (ECF) sigma factor

434 protein family. Molecular Microbiology 74: 557-581.

435 Tzung SP, Kim KM, Basañez G, Giedt CD, Simon J, Zimmerberg J, Zhang KYJ and Hockenbery

436 DM (2001). Antimycin A mimics a cell-death-inducing Bcl-2 homology domain 3. Nature Cell 
437 Biology 3: 183-191.

438 Yan Y, Zhang L, Ito T, Qu X, Asakawa Y, Awakawa T, Abe I and Liu W. (2012). Biosynthetic 439 Pathway for High Structural Diversity of a Common Dilactone Core in Antimycin Production.

440 Organic Letters 14: 4142-5.

441 


\section{Figure 1}

The antimycin biosynthetic gene cluster in Streptomyces albus S4.

Genes shaded grey indicate those that are required for antimycin biosynthesis. Genes shaded black were experimentally determined not to be required for antimycin biosynthesis. Narrow black arrows indicate the presence of four operons and the direction of their transcription. The locations of cosmid 213 and cosmid 456 are indicated by horizontal lines and the double vertical hash indicates that cosmid 456 is comprised of additional DNA that falls outside the boundaries of this schematic.

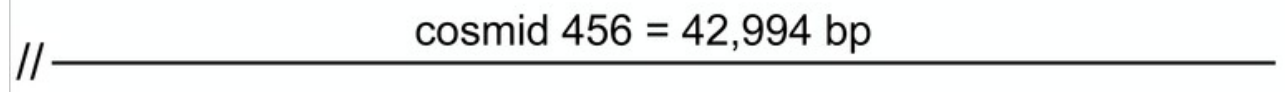

cosmid $213=35,797 \mathrm{bp}$

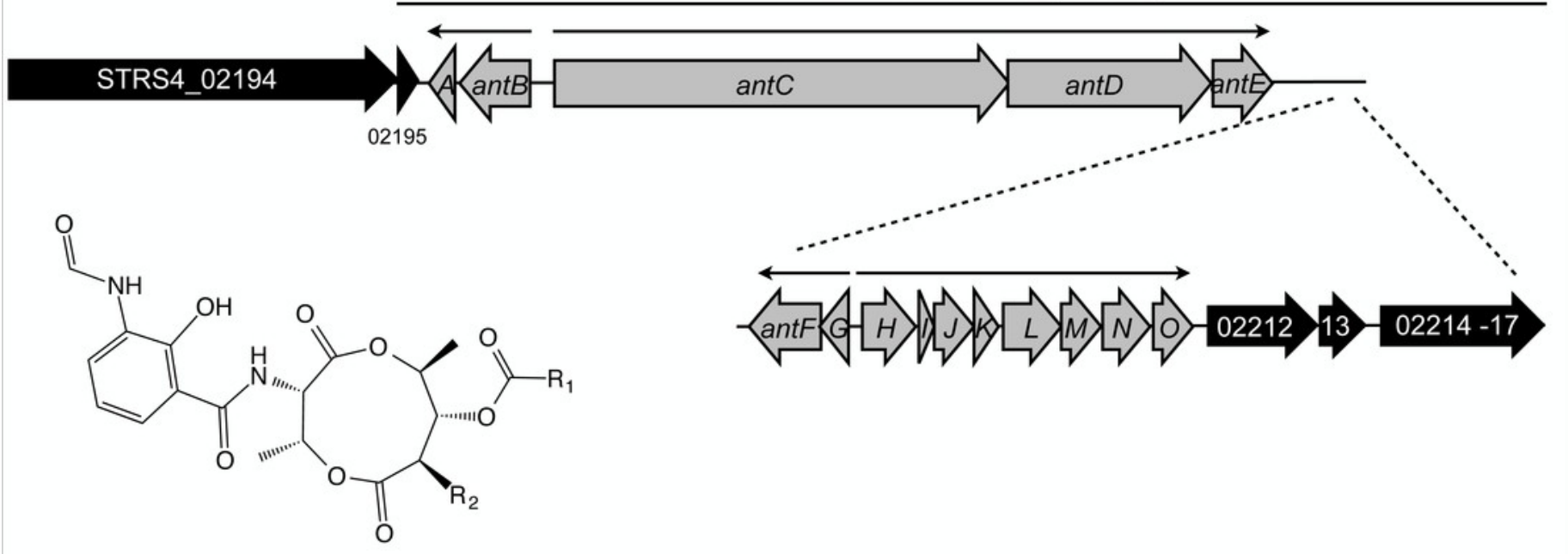

Antimycin $\mathrm{A}_{1} \mathrm{R}_{1}=\mathrm{CH}\left(\mathrm{CH}_{3}\right) \mathrm{CH}_{2} \mathrm{CH}_{3}, \mathrm{R}_{2}=\left(\mathrm{CH}_{2}\right)_{5} \mathrm{CH}_{3}$

Antimycin $\mathrm{A}_{2} \mathrm{R}_{1}=\mathrm{CH}\left(\mathrm{CH}_{3}\right)_{2}, \mathrm{R}_{2}=\left(\mathrm{CH}_{2}\right)_{5} \mathrm{CH}_{3}$

Antimycin $\mathrm{A}_{3} \mathrm{R}_{1}=\mathrm{CH}\left(\mathrm{CH}_{3}\right) \mathrm{CH}_{2} \mathrm{CH}_{3}, \mathrm{R}_{2}=\left(\mathrm{CH}_{2}\right)_{3} \mathrm{CH}_{3}$

Antimycin $\mathrm{A}_{4} \mathrm{R}_{1}=\mathrm{CH}\left(\mathrm{CH}_{3}\right)_{2}, \mathrm{R}_{2}=\left(\mathrm{CH}_{2}\right)_{3} \mathrm{CH}_{3}$ 


\section{Figure 2}

Defining the boundaries of the antimycin gene cluster.

(A) Streptomyces albus S4 WT and mutant strains challenged with Candida albicans. Null mutants of genes adjacent to the gene cluster (coloured black in Fig. 1) produce an antimycin-positive phenotype, characterised by a large circular zone of cleared C. albicans growth. The $\triangle a n t C$ mutant strain displays an antimycin-negative phenotype, but retains residual antagonistic activity against $C$. albicans due to the production of candicidin, a second antifungal compound produced by this strain (Barke et al., 2010; Seipke et al., 2011a). (B) High-performance liquid chromatography (HPLC) of metabolites produced by S. albus S4 WT and mutant strains. The $\triangle a n t C$ mutant does not produce antimycins, while null mutations in genes adjacent to the antimycin cluster had no effect on antimycin production.

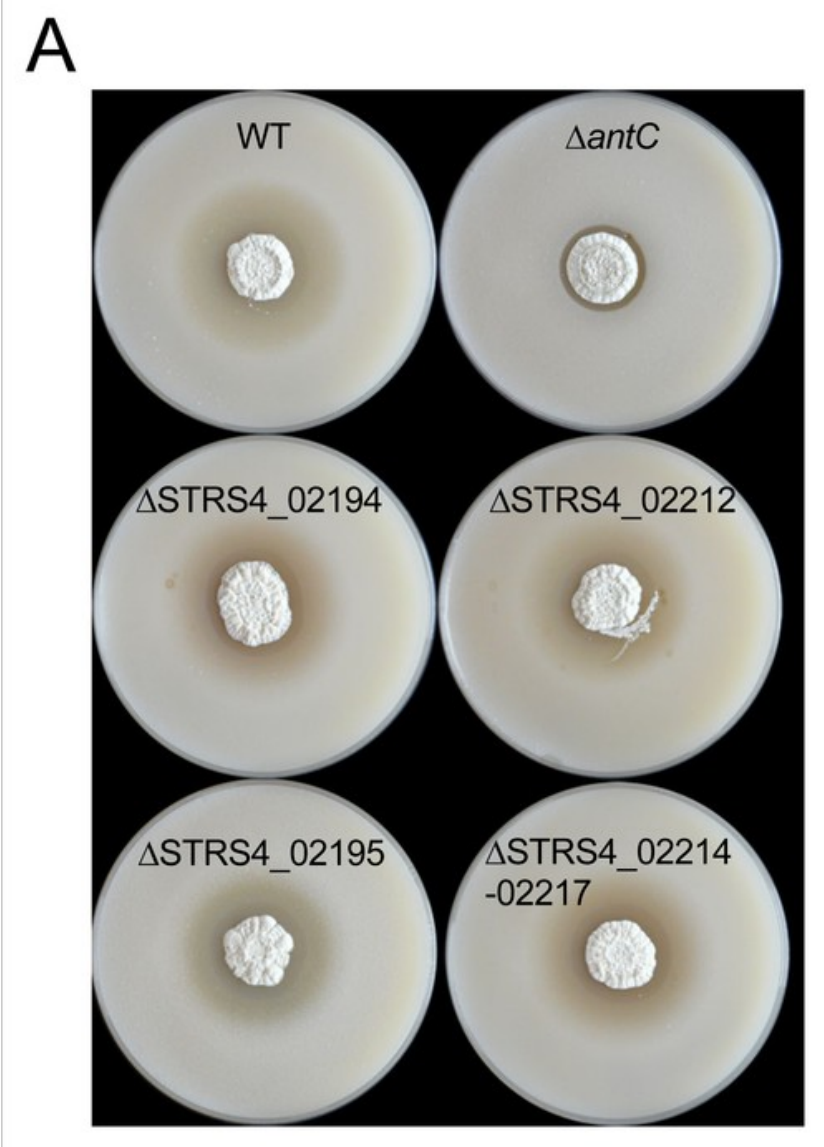

B

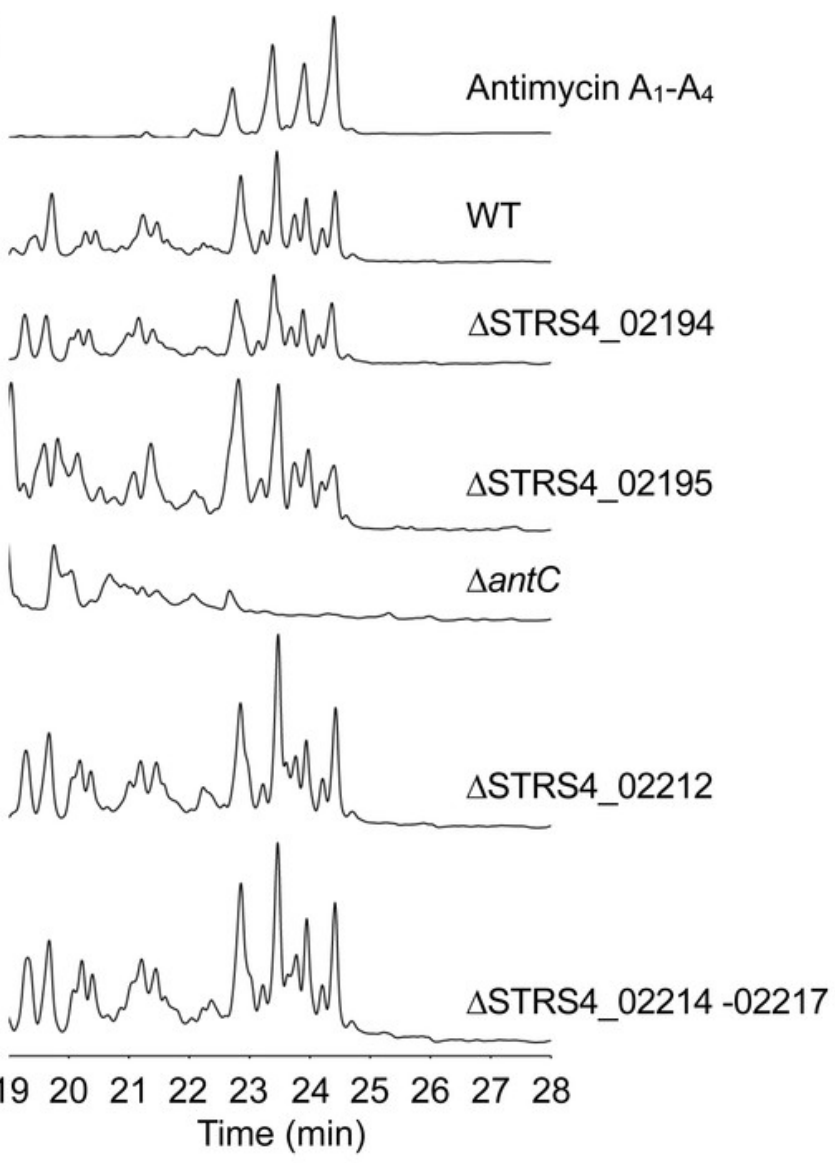




\section{Figure 3}

There is a delay between expression of the antimycin biosynthetic genes and the production of antimycins.

(A) HPLC analysis of metabolites produced by S. albus S4 wild-type. Antimycins are detected in media extracts of 42 hour old but not 18 hour old cultures. (B) qRT-PCR analysis of the antimycin gene cluster in 18 and 42 hour old cultures shows that expression of the antimycin gene cluster is significantly down-regulated following differentiation. ${ }^{* * *}$ denote that values reported are statistically significantly different with a $P$ value $<0.001$ in a Student's T-test.

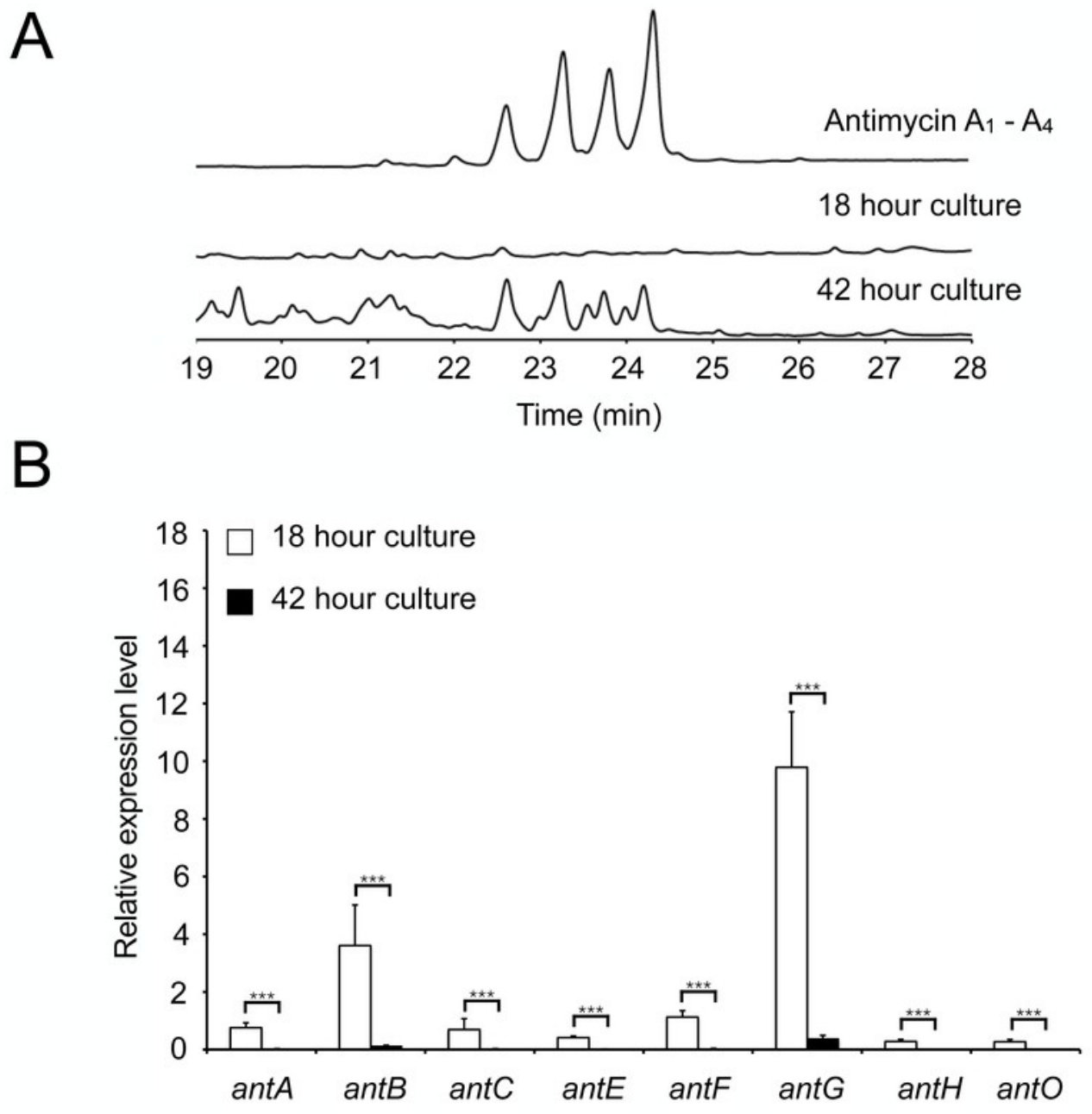




\section{Figure 4}

$\sigma^{\text {AntA }}$ is required for the biosynthesis of antimycins.

(A) S. albus S4 strains challenged with Candida albicans. The $\triangle a n t A$ null mutant shows dramatically reduced bioactivity compared to the wild-type strain and the complemented strain ( $\triangle a n t A / p l J 10257-a n t A)$. The residual bioactivity of the $\triangle a n t A$ mutant is due to the continued production of candicidin, a second antifungal compound. (B) HPLC analysis of metabolites produced by S. albus S4 strains. Antimycins were only detected in extracts prepared from the wild-type and the $\triangle a n t A / p l J 10257$-antA strains, and not $\triangle a n t A$ null mutant.

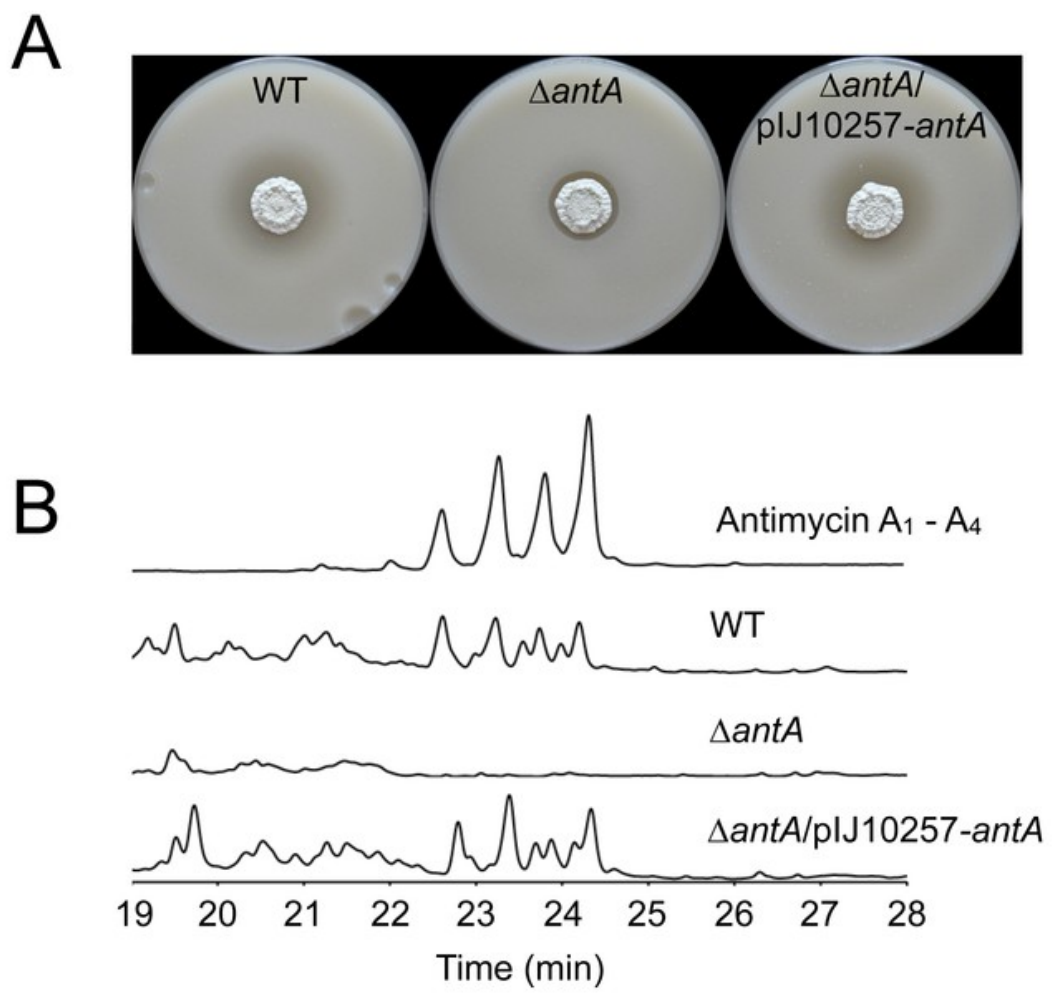




\section{Figure 5}

$\sigma^{\text {AntA }}$ activates transcription of the antFG and antHIJKLMNO operons.

qRT-PCR analysis of antimycin genes in the wild-type and $\triangle a n t A$ strains after 18 hours growth. Transcription of antFG and antHIJKLMNO is significantly reduced in the $\triangle a n t A$ mutant strain, whereas transcription of antBCDE are unaffected. ${ }^{* * *}$ denote that values reported are statistically significantly different in a Student's $t$ test with a $P$ value $<0.001$ in a Student's T-test.

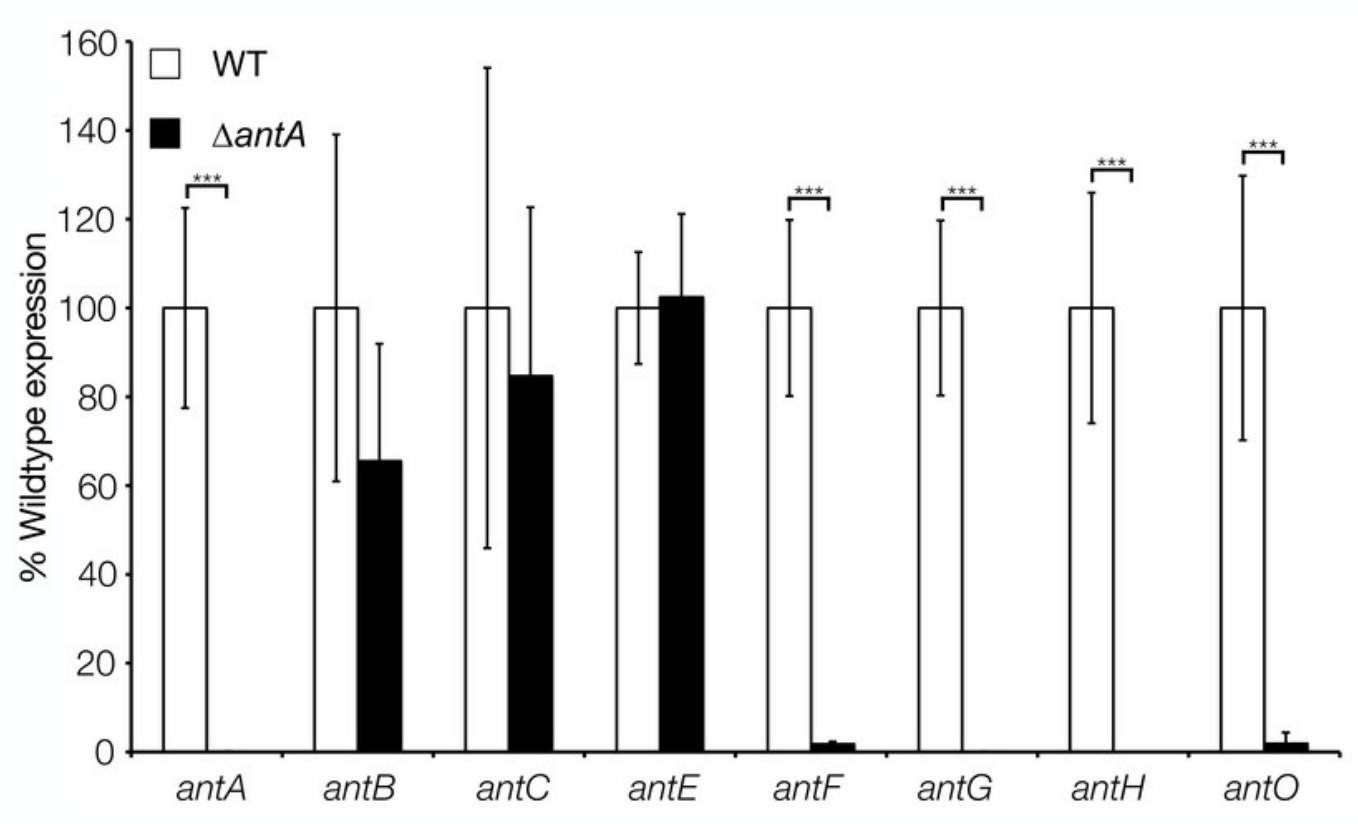




\section{Figure 6}

$\sigma^{A n t A}$ alone is sufficient to activate transcription of antFG and antHIJKLMNO in 42 hour old cultures.

qRT-PCR analysis of wild-type or $\triangle a n t A /$ plJ10257-antA in 42 hour old cultures shows that

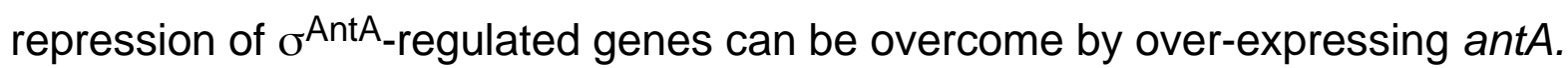

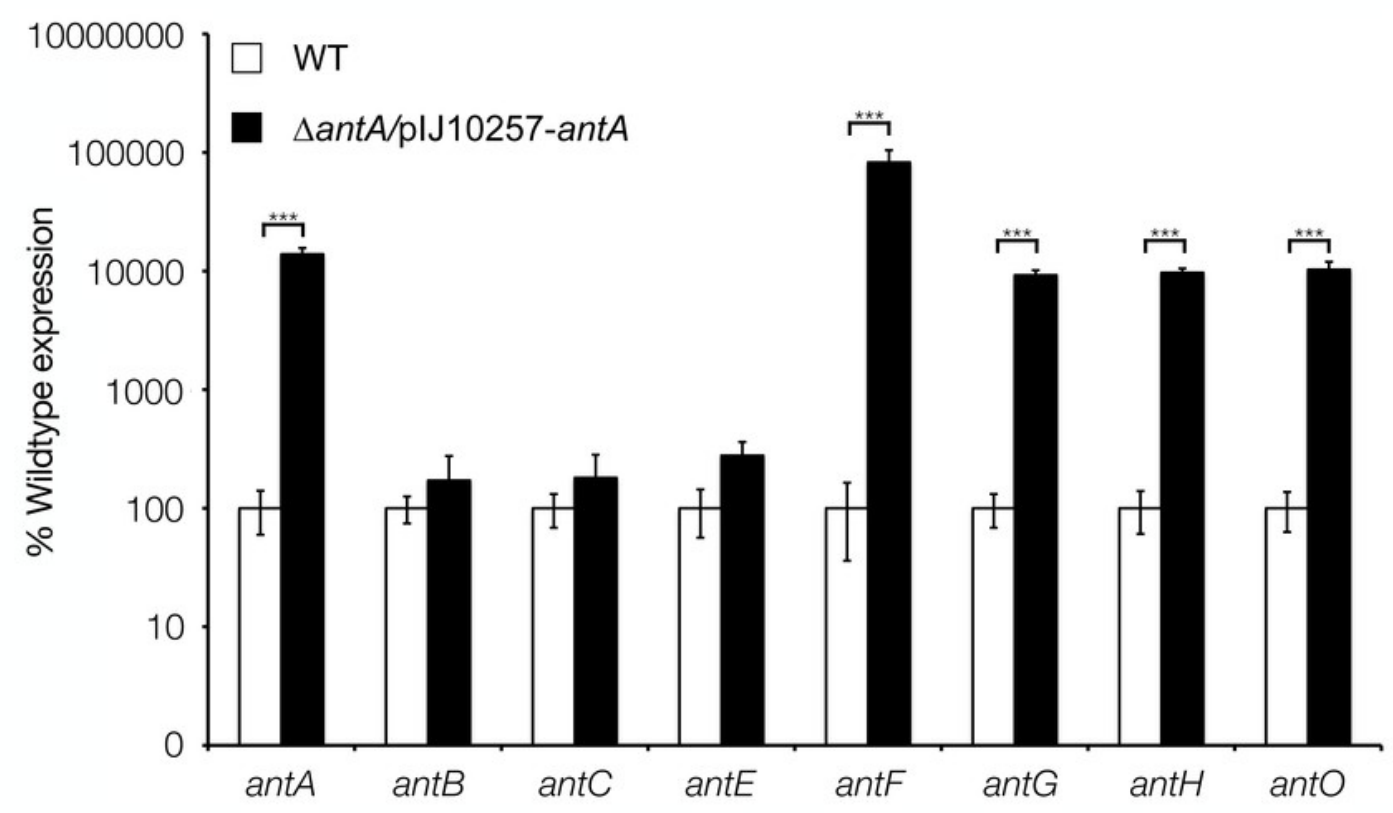




\section{Figure 7}

Identification of $\sigma^{\text {AntA }}$ promoter motifs.

(A) The -10 and -35 motifs at the $\sigma^{A n t A}$-target promoters of antFG and antHIJKLMNO are nearly $100 \%$ identical and display zero nucleotide identity with the promoter region of antAB. Shared identity is indicated by grey shading. The nucleotides mapped by 5'RLM-RACE is denoted by +1 and are shown in bold face (B) Conservation of the S. albus S4 antFG and antHIJKLMNO promoter elements in other antimycin-producing Streptomyces species. Conservation between the experimentally determined promoter region of $S$. albus S4 and the putative promoter regions of other antimycin producers is indicated by grey shading. (C) Consensus sequence for the -35 and -10 promoter elements recognised by $\sigma^{\text {AntA }}$ displayed as a WebLogo (Crooks et al., 2004). Below are the full strain names and accession numbers for antimycin-producing strains: S. ambofaciens ATCC 23877 (AM238663), S. blastmyceticus NBRC 12747 (AB727666), S. gancidicus BKS 13-15 (AOHP00000000), S. griseoflavus Tü4000 (ACFA00000000), S. hygroscopicus subsp. jinggangensis 5008 (NC_017765), S. hygroscopicus subsp. jinggangensis TL01 (NC_020895), Streptomyces sp. 303MFCol5.2 (ARTR00000000), Streptomyces sp. TOR3209 (AGNH00000000), S. albus S4 (CADY00000000), S. albus J1074 (NC_020990), Streptomyces sp. SM8 (AMPN00000000), Streptomyces sp. NRRL2288 (JX131329), Streptomyces sp. LaPpAH-202 (ARDM00000000), Streptomyces sp. CNY228 (ARIN00000000). 


\section{A \\ S. albus $S 4$ promoters mapped by $5{ }^{\prime} R L M-R A C E$}

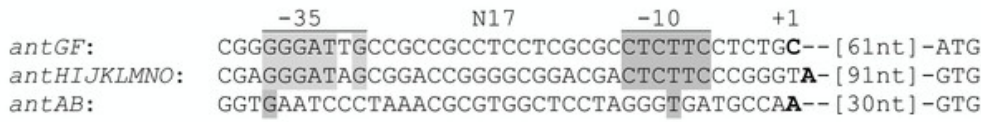

\section{B Predicted $\sigma^{\text {AntA }}$ binding sites in other antimycin producers}

\section{antFG promoter}
S. albus 54
S. albus $\mathrm{J} 1074$
S. SM8
S. NRRL 2288
S. LaPpAH-202
S. CNY228
S. ambofaciens
S. 303MFCo 13.2
S. gancidicus
S. TOR3209
S. hygroscopicus TLO1
S. hygroscopicus 5008
S. griseoflavus
S. blastmyceticus

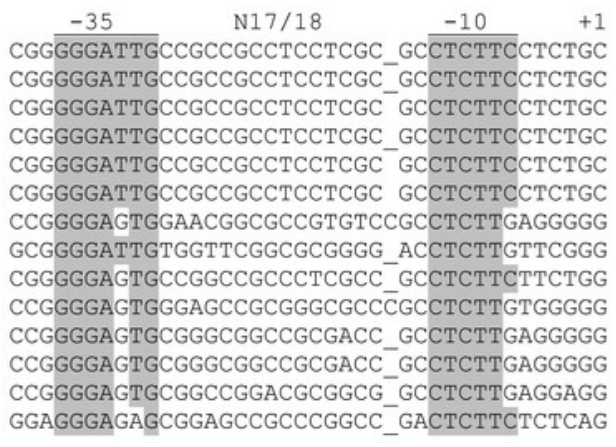

antHIJKLMNO promoter

$\begin{array}{llll}-35 & \mathrm{~N} 17 / 18+10 \quad+1\end{array}$ CGAGGGATAGCGGACCGGGGCGGACGA CTCTTCCCGGGTA CGGGGGATAGCGGACCGGGCCGGACGA_CTCTTCCCGGGTA CGGGGGATAGCGGACCGGGCCGGACGA_CTCTTCCCGGGTA CGAGGGATAGCGGACCGGGGCGGACCA СTCTTCCCGGGTA CGAGGGATAGCGGACCGGGGCGGACGA_CTCTTCCCGGGTA CGAGGGATAGCGGACCGGGGCGGACGA_CTCTTCCCGGGTA ACGGGGATAGCCGGGCCGCCCGCCCGGCCTCTTACCGCAAC CGGGGGATTGCGGGTCTCCCGCACGGC CTCTTTCCCAGAC GCGGGGATAGCGGGCGCGGTGCGCGGC_CTCTGTTCTCGGT GCGGGGATAGCCGGACCGTCCGTCCGGCCTCTTACCGCAAC GCAGGGATAGCCGGGCCGCCCGCCCGGCCTCCTTACCGGAA GCAGGGATAGCCGGGCCGCCCGCCCGGCCTCCTTACCGGAA CCGGGGATAGCCGGGCCGCACGCCCGGCCTCTTACCGCAAC GCGGGGATGGAACTCCCCTGCCCCGTA CTCCTTATGTCTG

\section{C} -35 consensus
-10 consensus
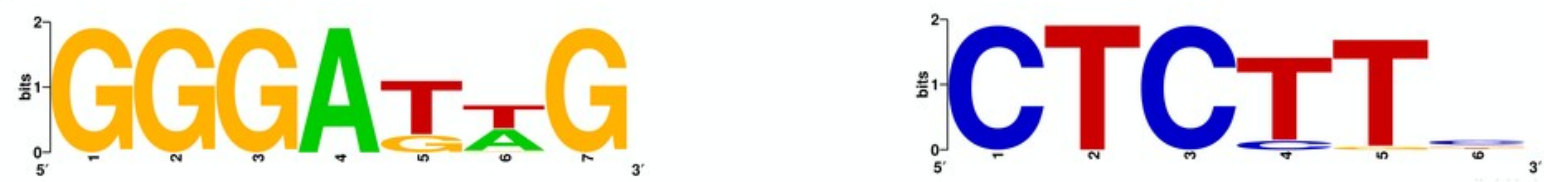


\section{Figure 8}

Altering the terminal Ala-Ala motif of $\sigma^{\text {AntA }}$ results in higher expression of $\sigma^{\text {AntA }}$ targets.

The $\triangle a n t A$ null mutant was complemented with either a wild-type copy of ant $A$ or a variant of antA encoding A172D and A173D point mutations. After 42 hours of growth, transcription of $\sigma^{A n t A}$-targets in the $\triangle a n t A / a n t A-D D$ strain were significantly greater than both the wild-type and $\triangle a n t A / a n t A-A A$ strains, suggesting the terminal Ala-Ala motif modulates stability and/or activity of $\sigma^{\text {AntA }}{ }^{* *}$ and ${ }^{* * *}$ denote that values reported are statistically significantly different with a $P$ value $<0.01$ and 0.001 in a Student's T-test, respectively.

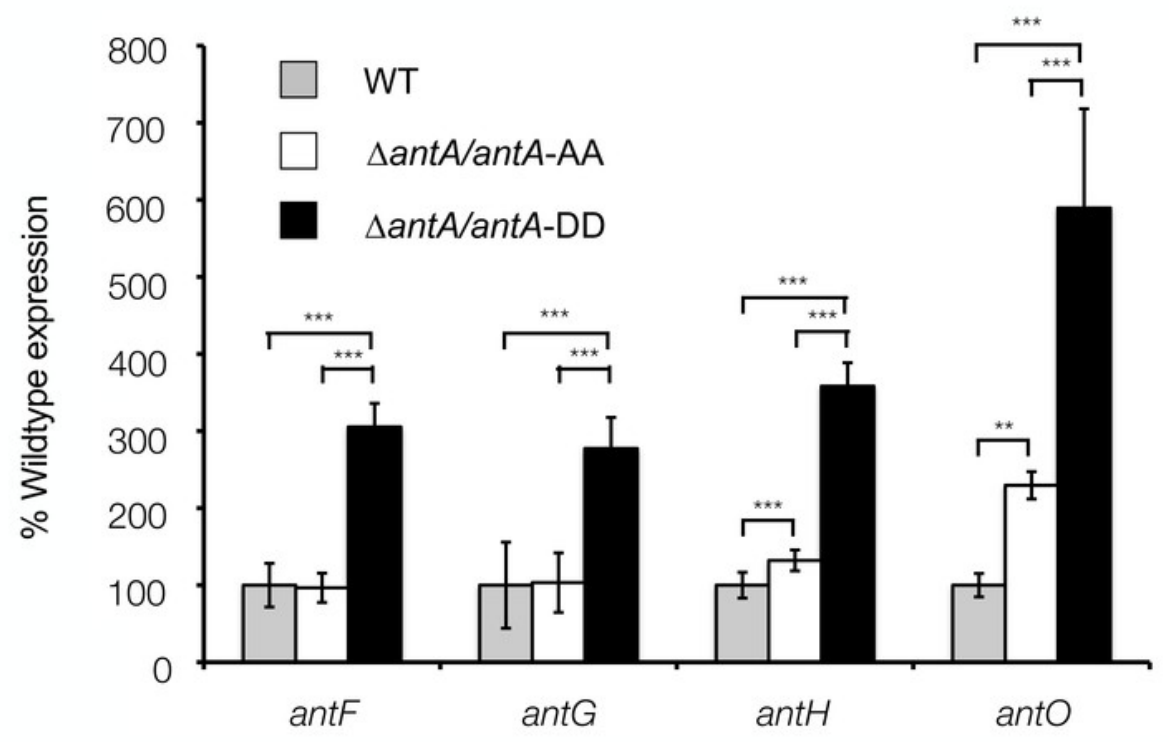

